\title{
Mitochondrial DNA as a potential tool for early cancer detection
}

\author{
Ryan L. Parr, '* Gabriel D. Dakubo,' Robert E. Thayer,' Keith McKenney² and Mark A. Birch-Machin ${ }^{3}$ \\ I Genesis Genomics Inc, 1294 Balmoral Street, Thunder Bay, Ontario, P7B 5Z5, Canada \\ ${ }^{2}$ School of Computational Sciences, George Mason University, GMU-PW, MS 4E3 10900 University Blvd., Manassas, VA 20110, USA \\ ${ }^{3}$ Dermatological Sciences, University of Newcastle, Newcastle upon Tyne, NE2 4HH, UK \\ * Correspondence to: Tel.: + 1807346 8100; Fax: +1 807346 8105; E-mail: Ryan.Parr@genesisgenomics.com
}

Date received: 14th September 2005

\begin{abstract}
The recent surge in mitochondrial research has been driven by the identification of mitochondria-associated diseases and the role of mitochondria in apoptosis. Both of these aspects have identified mitochondrial analysis as a vital component of medical research. Moreover, mitochondria have been implicated in the process of carcinogenesis because of their vital role in energy production, nuclear-cytoplasmic signal integration and control of metabolic pathways. Interestingly, at some point during neoplastic transformation, there is an increase in reactive oxygen species, which damage the mitochondrial genome. This accelerates the somatic mutation rate of mitochondrial DNA. It has been proposed that these mutations may serve as an early indication of potential cancer development and may represent a means for tracking tumour progression. The purpose of this review is to explore the potential utility that these mutations may afford for the identification and monitoring of neoplasia and malignant transformation where appropriate body fluids or non-invasive tissue access is available for mitochondrial DNA recovery. Specifically, prostate, breast, colorectal, skin and lung cancers are discussed.
\end{abstract}

Keywords: mitochondrial DNA (mtDNA), mutations, polymorphism, cancers, homoplasmy, heteroplasmy, metabolism, biomarker, reactive oxygen species (ROS), early detection

\section{Introduction}

\section{Mitochondrial characteristics}

There are several biological characteristics which cast mitochondria and, in particular, the mitochondrial genome, as a biological tool for early detection and monitoring of neoplasia and its potential progression. These vital characteristics are important in cancer research, as not all neoplasias become malignant. ${ }^{1}$ Mitochondria are archived in the cytoplasm of the ovum and as such do not recombine. ${ }^{2}$ This genome has an accelerated mutation rate, by comparison with the nucleus, ${ }^{3}$ and accrues somatic mutations in tumour tissue. ${ }^{4}$ Moreover, mitochondrial DNA (mtDNA) has a high copy number in comparison with the nuclear archive of DNA. There are potentially thousands of mitochondrial genomes per cell, which enables detection of important biomarkers, even at low levels. In addition, mtDNA can be heteroplasmic, which means that disease-associated mutations occur in a subset of the genomes. The presence of heteroplasmy is an indication of disease and is found in many human tumours. ${ }^{5,6}$ Identification of low levels of heteroplasmy may allow unprecedented early identification and monitoring of neoplastic progression to malignancy. Mitochondria have been implicated in the carcinogenic process because of their role in apoptosis ${ }^{7}$ and other aspects of tumour biology. Deletions in the mitochondrial genome are associated with organelle dysfunction and serve as biomarkers as well. ${ }^{8}$ Finally, mtDNA is a modest molecule by comparison with its nuclear cellmate. The compact size of the mitochondrial genome allows extensive, economical biological characterisation such as complete genome sequencing. Coding for just 13 enzyme complex subunits, 22 transfer RNAs and two ribosomal RNAs, the mitochondrial genome is packaged in a compact 16,569 base pair (bp) circular molecule. ${ }^{9}$ These products participate in the critical electron transport process of ATP production. Collectively, mitochondria generate 80 per cent of the chemical fuel which fires cellular metabolism. As a result, nuclear investment in the mitochondria is high that is, several thousand nuclear genes control this organelle in order to accomplish the complex interactions required to maintain a network of pathways, which coordinate energy demand and supply.

\section{Prostate cancer}

Prostate cancer $(\mathrm{PCa})$ is a transformation of the secretory epithelial cells lining the ductal network of the prostate gland. 
In the USA alone, over 230,000 men developed PCa in 2005 (Facts and Figures, American Cancer Society, 2005). The way in which human prostate epithelial cells metabolise citrate is strikingly different from other soft tissues.

The peripheral zone of the prostate gland, which accounts for the majority of prostate tumours, has a uniquely different metabolism. For this reason, the metabolic characteristics of this tissue are central to mtDNA damage and its role in PCa. Unlike the central zone, the peripheral zone cells accumulate very high levels of zinc, due in part to the activity of a zinc transporter. ${ }^{10,11}$ Zinc levels in these cells are about ten-fold higher than in other cell types. The high zinc levels in the mitochondria inhibit an initial Krebs cycle enzyme, aconitase, thereby preventing the isomerisation of citrate to isocitrate, which is required for the complete metabolism of citrate. This precludes the movement of $\mathrm{NADH}$ and $\mathrm{FADH}_{2}$ from the Krebs cycle into the electron transport chain. Interestingly, during the pathway of malignant transformation, zinc levels drop and the Krebs cycle becomes active. Peripheral zone prostate cells then become energy efficient, consuming substantial amounts of oxygen, in contrast to many other tumours that are energy inefficient. The increased functional capacity of the Krebs cycle in these transforming cells might be responsible for the up-regulation of enzymes involved in energy-generating pathways such as $\beta$-oxidation. ${ }^{12,13}$

Hypothetically, this raises the intriguing possibility of high reactive oxygen species (ROS) production, which can cause significant oxidative damage to the mitochondrial genome. There may be a sudden increase, or burst, of ROS, which heavily damage mtDNA, perhaps overwhelming any operational mtDNA repair mechanisms. Proteomics analysis reveals altered expression of nuclear-encoded complex IV subunits in pre-malignant prostate epithelial cells. ${ }^{14}$ Such protein changes could affect the functions of this complex, resulting in defective oxidative phosphorylation. Thus, a state of reversed electron flow in the redox series can arise, leading to elevated ROS production. Under normal circumstances, elevated ROS induces the activity of antioxidant scavengers such as glutathione peroxidase. In support of the pivotal role of ROS in PCa development, however, is the early silencing of the gene encoding glutathione S-transferase- $\pi$ (GSTP1) via hypermethylation of its promoter in $\mathrm{PCa}^{15}$ and the presence of high levels of hydrogen peroxide in PCa cells compared with normal prostate epithelial cells. ${ }^{16}$

An early metabolic switch which may lead to increased ROS production in association with a loss of antioxidant mechanisms should culminate in accelerated mtDNA mutations in PCa. Not surprisingly then, the few reports of mtDNA mutations in PCa indicate a state of hypermutagenesis. ${ }^{17-20}$ The first study of mtDNA mutations in PCa examined large mtDNA deletions by gel electrophoresis and observed a positive correlation between deletion frequency and advancing age. ${ }^{21}$ Four other studies involved sequencing regions of the mtDNA genome. The D-loop and adjacent regions were examined in three studies. ${ }^{17-19}$ In these studies, a total of 34 substitutions and four small insertions and deletions were detected. In addition, the following mutations were detected in coding genes flanking the D-loop: one mutation each in the $16 \mathrm{~S}$ ribosomal RNA gene $(16 \operatorname{SrRN} A)$ and the transfer RNA leucine gene $\left(t R N A^{\text {leu }}\right)$; three in $N D 4$; and four each in ND1, ND5 and CYTB. The fifth and most recent study of mtDNA alterations in PCa focused on the COI gene. This study involved a large population sample from which a total of 21 unique nucleotide changes were detected in PCa. Two mutations, G6261A (six individuals) and A6663G (five individuals) seem to be mutation hot spots in PCa.

MtDNA alterations in the prostate gland may have significant utility for early PCa detection, given the uniqueness of this molecule and the mode of occurrence of mtDNA mutations in the prostate. The metabolic and mtDNA alterations in the prostate are likely to be early events in PCa development, since they are observed in pre-malignant histological benign-appearing glands. Prostate epithelial cells are present in prostate fluids such as prostate massage fluid, and the high copy number of mitochondrial genomes coupled with the accelerated mtDNA mutations in PCa and, in some instances, the homoplasmic nature of the mutations, should offer a detection advantage over nuclear genome alterations in biofluids. ${ }^{19,22}$

\section{Breast cancer}

Much like prostate cancer, breast cancer is also a transformation of the epithelial secretory cells lining the mammary ducts. Breast cancer was diagnosed in nearly 213,000 women in the USA in 2005 (Facts and Figures, American Cancer Society, 2005). More than ten papers describing altered mtDNA in human breast tumour tissue have been published in the two years since Carew and Huang published their comprehensive review of mtDNA defects in cancer. ${ }^{4}$ There have been a variety of mtDNA alterations found in breast tumour tissue since the first report published by Bianchi et al. about ten years ago. ${ }^{23}$ While the precise frequency, region of the mtDNA genome characterised and nature of mtDNA mutations associated with breast tumour tissue varies in each report, there is compelling evidence that somatic mutations in mtDNA represent informative biomarkers for detecting disease. A number of investigators successfully obtained sufficient quantities of DNA from nipple aspirate fluid (NAF) and from ductal lavage to perform mtDNA analysis, thus providing the possibility of non-invasive methods using mtDNA as a sensitive means to detect breast cancer.

Rates of somatic mtDNA mutations in breast cancer range from that reported in a 2002 study by Tan et al. ${ }^{24}$ (74 per cent; $14 / 19$ tested) to the recent results of Tan et al. ${ }^{25}$ looking at a much larger sample size, with a reported rate of 45 per cent heteroplasmy. Zhu et al. found mutated 
mtDNA in 93 per cent of breast cancer samples (14 of 15 tested), with the frequency of mutations higher in the D-loop than in other loci tested ( 1.5 per cent versus 0.18 per cent). ${ }^{26}$ In addition, Zhu et al. tested whether NAF provided mtDNA that carried mutations found in the patient's tumour and found that 40 per cent of the NAF samples contained some of the mutations observed in the patient's tumour mtDNA. Rosson and Keshgegian found mtDNA mutations in 15 of 15 tumour samples micro-dissected by laser capture microscopy from 15 different patients. ${ }^{27}$ The number of mutations found in the D-loop ranged from one to 15 relative to an adjacent normal tissue control mtDNA sequence. As others have found that adjacent normal control tissue does not necessarily represent the 'wild-type' and frequently harbours tumour-type mtDNA mutations, the results of Rosson and Keshgegian may under-report the true number of mutations in the samples studied. ${ }^{27}$ Zhu et al. found a 4,576 bp deletion in mtDNA to be a good marker for breast cancer, in that it was present 77 per cent of the time in 39 tumours and 0 per cent in true normal samples. ${ }^{28}$ Interestingly, 13 per cent of the histologically 'normal' adjacent tumour tissue samples showed the 4,576 bp deletion, suggesting that, while the histology appears normal, the genotype is moving towards the tumour state. Dani et al. found a different mtDNA deletion - the 4,977 base pair deletion - in 24 per cent of breast tumours but concluded that this resulted from contaminating adjacent tissue and, in fact, stated that tumours are essentially free of the 4,977 base pair deletion. ${ }^{29}$ This is in contrast to Zhu et al., who found the 4,977 base pair deletions in breast tumours (18/39, 46 per cent) to occur at a rate higher than in adjacent normal tissue $\left(13 / 39,33\right.$ per cent) and normal tissue $\left(6 / 23,26\right.$ per cent). ${ }^{28}$ The explanation for this apparent discrepancy remains to be determined. Isaacs et al. investigated the D310 ${ }^{30} \mathrm{mtDNA}$ marker in conjunction with loss of heterozygosity from 14 women of known BRCA1 status and found three of nine mtDNA mutations in BRCA1 carriers. They further demonstrated that the same DNA typing results could be obtained from NAF as well as ductal lavage samples. ${ }^{31}$ D310 is of particular note because in some groups this nucleotide is a $\mathrm{T}$ as opposed to a C. In a similar study, Parrella et al. looked at the D310 mtDNA marker and found that three of 20 mtDNA mutations (15 per cent) had mutated in the tumour compared with the normal tissue. ${ }^{32}$ They reported that, comparing tumour with blood mtDNA, 61 per cent of the tumours had mtDNA mutations.

\section{Colorectal cancer}

Colorectal cancer affected over 145,000 people in the USA alone in 2005 (Cancer Facts and Figures 2005). More than 95 per cent of colorectal cancers are adenocarcinomas which develop from adenomatous polyps (adenomas); however, only a small portion of these lesions progress to invasive cancer $(1-10$ per cent). Current diagnostic tools include the faecal occult blood test and the multitarget assay panel (MTAP), which looks for mutations associated with the nuclear genome (Jani National Cancer Institute). Deficiencies in DNA repair and a higher mutation rate in the mitochondria result in the accumulation of mutations that may provide a means for the early detection of human colorectal lesions (polyps or cancer) and may increase the utility of the MTAP test. For those at risk (familial or age related), a colonoscopy is the screening method of choice.

Previous research examining the D-loop or non-coding region (NCR), or performing complete sequencing of the mitochondrial genome in colorectal tumours, found that the majority of the somatic mutations were homoplasmic transitions and single bp insertions or deletions, and that there were few large-scale deletions. ${ }^{33-36}$ Habano et al. were among the first to report microsatellite instability in 20/45 colorectal cancer samples in the NCR of the mitochondrial genome. ${ }^{37}$ The nature of these alterations is consistent with ROS damage and low levels of DNA repair. Furthermore, somatic mutations and altered gene expression were found in: $12 \operatorname{SrRN} A ; 16 \operatorname{SrRNA}$; the NADH ubiquinone oxidoreductase subunit genes ND1, ND4, ND5; the cytochrome oxidase subunit genes COXI, COXII, COXIII; and the cytochrome gene CYTB. Recently, Guleng et al. reported no mutations in the ND1 and ND5 genes. ${ }^{38}$ Aikhionbare et al. investigated the mitochondrial genome with restriction analyses, followed by the sequencing of length variants for three histological subtypes of colorectal adenomas, in association with tumour and matched surrounding normal tissues. ${ }^{36}$ Thirty-eight sequence variants for the precursor and cancerous lesions were identified. This method does not detect single nucleotide polymorphisms (SNPs) that do not create a restriction site, however, meaning that the actual number of alterations could be higher. Interestingly, none of their 38 sequence variants matched the somatic mutations cited in previous reviews, ${ }^{4,5}$ but the regions of mitochondrial instability were similar. The results of Aikhionbare et al. indicate a high prevalence of mutations in tumour tissue, with much lower numbers in pre-cancerous lesions and no mutations in the surrounding normal tissue. A progressive pattern is indicated.

\section{Skin cancer}

The incidence of skin cancer has steadily increased over the past decade, with more than 1 million new cases currently being diagnosed each year in the USA and 65,000 in the UK (figure provided by Cancer Research UK). Apart from malignant melanoma, non-melanoma skin cancer (NMSC) accounts for around 90 per cent of skin cancers and consists of basal cell and squamous cell carcinomas (BCC and SCC, respectively). BCCs are the most common form of NMSC and arise predominantly from the basal keratinocytes of the epidermis but also from cells in hair follicles and sebaceous 
glands. They are locally invasive but rarely metastasise. SCCs are also derived from basal keratinocytes; however, in contrast to BCCs, SCCs may metastasise. Cutaneous malignant melanoma is a malignant tumour of the melanocytes, usually arising in the epidermis, and is the most lethal of the skin cancers.

The cause of the great majority of skin cancers, including malignant melanoma, in pale-skinned individuals is a combination of the DNA-damaging effects of ultraviolet radiation (UVR) in sunlight and the characteristics that determine the response of the body to that radiation. In human skin, mtDNA, rather than nuclear DNA, is a useful biomarker of UV-induced DNA damage. ${ }^{39-41}$ Moreover, within the field of skin cancer research, there is an additional advantage of using mtDNA, namely that there is no evidence of nuclear excision for the repair of DNA photoproducts (e.g. cyclobutane pyrimidine dimers) in mtDNA. ${ }^{42-45}$ Together, these factors lead to accumulation of photodamage in skin mtDNA without compromising cell function.

These aspects, together with the fact that UVR is important in the development of skin cancer and has been shown to induce mtDNA damage in human skin, ${ }^{40,46}$ led to the first detailed study of mtDNA damage in human NMSC. ${ }^{47}$ This work showed clear differences in the distribution of deletions between the tumour and the histologically normal perilesional skin.

In the literature, there are three recent studies that have investigated the relationship between mtDNA and cutaneous malignant melanoma. ${ }^{48-50}$ Two of the three studies ${ }^{48,49}$ have screened for mtDNA mutations (but not deletions or duplications) in the NCR alone, which constitutes less than 7 per cent of the mitochondrial genome. The study by Takeuchi et al. ${ }^{48}$ found that the somatic mutations in the D-loop region of tumour and paired plasma did not correlate with the clinicopathological characteristics. The study by Deichmann et al. ${ }^{49}$ found microsatellite instability (mtMSI) of mtDNA $\mathrm{C}_{n}$ tracts in the primary tumours. The larger patient study by Poetsch et al. ${ }^{50}$ investigated the D-loop, as well as looking for the $4,977 \mathrm{bp}$ common deletion in 61 primary malignant melanomas and in neighbouring normal skin tissue. Point mutations were a rare feature, whereas mtDNA instability in the D-loop (mtMSI) was found in 13 per cent of primary nodular tumours and in 20 per cent of metastases. The 4,977 bp common deletion was demonstrated in 10 per cent of melanomas.

The increased frequency of the mtDNA deletions and point mutations in human skin with increasing UVR exposure provides a measure of cumulative UV exposure in human skin. Together with the mtDNA damage identified in the skin cancer studies, this may in turn provide an early detection tool for skin cancer development, as well as providing a method for monitoring the long-term safety of clinical UV phototherapy regimens, since the risk of skin cancer must be minimised. Interestingly, mtDNA D-loop alterations can be detected in blood and may therefore potentially act as a circulating DNA melanoma marker. ${ }^{48}$ In addition, $\mathrm{C}_{\mathrm{n}}$ tract alterations occurred in two out of nine primary melanomas with subsequent metastasis, whereas none of the 20 nonmetastasising cutaneous melanomas were affected. ${ }^{49}$ These somatic changes may reflect genomic imbalance during tumour progression and may therefore be useful for the assessment of patients' prognosis with a simple, small punch biopsy. Early diagnosis of skin cancer is particularly desirable because of the successful methods of treatment which are currently available to the dermatologist.

\section{Lung cancer}

Lung cancer comprises a spectrum of diseases and is the leading cause of cancer-related deaths among men and women in developed countries. There are two major classifications of lung cancer, small cell (SCLC) and non-small cell (NSCLC) carcinoma. SCLC is divided into three subtypes: small cell, mixed small cell/large cell and combined small cell carcinoma (small cell lung cancer combined with neoplastic squamous and/or glandular components). Although SCLC is more responsive to chemotherapy and radiation, it is usually well spread at primary diagnosis, making it difficult to treat successfully. Even with treatment, the overall survival rate at five years is $5-10$ per cent. NSCLC is a conglomeration of at least three distinct pathohistologies: squamous cell carcinoma, adenocarcinoma and large cell carcinoma. Localised surgical resection is the standard treatment; however, all newly diagnosed NSCLC patients are possible candidates for treatment studies.

Currently, there are more than 170,000 single lung nodules 'discovered' in patients each year in the USA alone. (Facts and Figures, American Cancer Society, 2005) Most of these are found during routine lung X-rays, meaning that detection techniques are really based on chance. Confirmation of diagnosis includes a follow-up biopsy, computed tomography (CT) scan or 'watchful waiting', where X-ray or CT scans are repeated at intervals to assess changes in the lesion. The 'gold standard' is surgical resection; however, 60 per cent of lung nodules are benign, hence, over half of the patients will undergo unnecessary surgery. From a diagnostic perspective, there are promising molecular tools, such as those detecting telomerase activity in tumour cells.

Improvement in the diagnostic strategy for lung cancer is a critical need in oncology. Indeed, the high mortality and morbidity is due to diagnosis of the disease well after it has spread. MtDNA may offer an advantage over current techniques. Suzuki et al. scored D-loop mutations in 12 SCLC and 16 NSCLC matched B-lymphoblastoid cells lines. ${ }^{51}$ In addition, a subset of the $C_{n}$ was sequenced in 55 resected NSCLC and corresponding non-malignant lungs. Mutations were observed in 17 of the 28 cell lines (61 per cent; eight of 12 SCLC and nine of 16 NSCLC). Moreover, 95 per cent of the SNPs occurred in the hypervariable regions. In 
addition, single base substitutions were more frequent in the D-loop if the $\mathrm{C}_{\mathrm{n}}$ was affected by an insertion or a deletion. For this reason, only the $C_{n}$ was sequenced in the 55 resected samples. Within this group, 11 (20 per cent) had changes. Likewise, in an earlier study, Fliss et al. found a 43 per cent mutation rate in six of 14 lung cancer samples when sequencing the entire genome and D-loop only from selected samples. ${ }^{22}$ Further work may identify sputum as a reliable source of mtDNA for monitoring lung neoplasia.

\section{Conclusions}

There is a pressing need to focus on the treatment, and therefore the early identification and subsequent monitoring, of pre-cancerous lesions, ${ }^{1}$ and the analyses of changes in the mitochondrial genome, associated with neoplasia, holds promise in fulfilling this need. Here we have reviewed five specific types of cancer which have tissue, or biofluids, from which mtDNA can be extracted and analysed from an early detection perspective. Although promising, the early results described here await further work and validation. There are many important questions yet to be addressed: such as the relationship between mtDNA and the actual disease; are mutations causative or merely a reflection of nuclear instability? And, are these processes independent events? Alterations in the non-coding D-loop suggest genome instability; however, as studies focus more on the coding regions of the mitochondrial genome, particularly in the case of nonsynonymous mutations in the genes contributing products to the electron transport process, metabolic implications are evident. Moreover, mutations in mitochondrial transfer RNAs indicate the possibility of a global mitochondrial translational shut down. Importantly, the wide range in the number and location of mitochondrial alterations in patients, within and between studies addressing the same malignancies, indicates the need for a coordinated approach among researchers. Finally, the interplay between nuclear and mitochondrial genes requires careful investigation and may hold the final understanding of the mitochondrial role in tumourigenesis. ${ }^{52}$

\section{Acknowledgments}

We thank Dr Eleftherios Diamandis for providing critical comments on an earlier version of this manuscript. Genesis Genomics Inc supported this work.

\section{References}

1. O'Shaughnessy, J.A., Kelloff, G.J., Gordon, G.B. et al. (2002), 'Treatment and prevention of intraepithelial neoplasia: An important target for accelerated new agent development', Clin. Cancer Res. Vol. 8, pp. 314-346.

2. Olivo, P.D., Van de Walle, M.J., Laipis, P.J. and Hauswirth, W.W. (1983), 'Nucleotide sequence evidence for rapid genotypic shifts in the bovine mitochondrial DNA D-loop', Nature Vol. 306, pp. 400-402.
3. Brown, W.M., George, Jr, M. and Wilson, A.C. (1979), 'Rapid evolution of animal mitochondrial DNA', Proc. Natl. Acad. Sci. USA Vol. 76, pp. 1967-1971.

4. Carew, J.S. and Huang, P. (2002), 'Mitochondrial defects in cancer', Mol. Cancer Vol. 1, pp. 9.

5. Penta, J.S., Johnson, F.M., Wachsman, J.T. and Copeland, W.C. (2001), 'Mitochondrial DNA in human malignancy', Mutat. Res. Vol. 488, pp. 119-133.

6. Legros, F., Malka, F., Frachon, P. et al. (2004), 'Organization and dynamics of human mitochondrial DNA', J. Cell Sci. Vol. 117, pp. 2653-2662.

7. Kroemer, G. and Reed, J.C. (2000), 'Mitochondrial control of cell death', Nat. Med. Vol. 6, pp. 513-519.

8. Srivastava, S. and Moraes, C.T. (2005), 'Double-strand breaks of mouse muscle mtDNA promote large deletions similar to multiple mtDNA deletions in humans', Hum. Mol. Genet. Vol. 14, pp. 893-902.

9. Anderson, S., Bankier, A.T., Barrell, B.G. et al. (1981), 'Sequence and organization of the human mitochondrial genome', Nature Vol. 290, pp. $457-465$.

10. Costello, L.C. and Franklin, R.B. (2000), 'The intermediary metabolism of the prostate: A key to understanding the pathogenesis and progression of prostate malignancy', Oncology Vol. 59, pp. 269-282.

11. Costello, L.C., Feng, P., Milon, B. et al. (2004), 'Role of zinc in the pathogenesis and treatment of prostate cancer: Critical issues to resolve', Prostate Cancer Prostatic Dis. Vol. 7, pp. 111-117.

12. Luo, J., Zha, S., Gage, W.R. et al. (2002), 'Alpha-methylacyl-CoA racemase: A new molecular marker for prostate cancer', Cancer Res. Vol. 62, pp. 2220-2226.

13. Armand, R., Channon, J.Y., Kintner, J. et al. (2004), 'The effects of ethidium bromide induced loss of mitochondrial DNA on mitochondrial phenotype and ultrastructure in a human leukemia T-cell line (MOLT-4 cells)', Toxicol. Appl. Pharmacol. Vol. 196, pp. 68-79.

14. Herrmann, P.C., Gillespie, J.W., Charboneau, L. et al. (2003), 'Mitochondrial proteome: Altered cytochrome c oxidase subunit levels in prostate cancer', Proteomics Vol. 3, pp. 1801-1810.

15. Millar, D.S., Ow, K.K., Paul, C.L. et al. (1999), 'Detailed methylation analysis of the glutathione S-transferase pi (GSTP1) gene in prostate cancer', Oncogene Vol. 18, pp. 1313-1324.

16. Lim, S.D., Sun, C., Lambeth, J.D. et al. (2005), 'Increased Nox1 and hydrogen peroxide in prostate cancer', Prostate Vol. 62, pp. 200-207.

17. Chen, J.Z., Gokden, N., Greene, G.F. et al. (2003), 'Simultaneous generation of multiple mitochondrial DNA mutations in human prostate tumors suggests mitochondrial hyper-mutagenesis', Carcinogenesis Vol. 24, pp. 1481-1487.

18. Chen, J.Z., Gokden, N., Greene, G.F. et al. (2002), 'Extensive somatic mitochondrial mutations in primary prostate cancer using laser capture microdissection', Cancer Res. Vol. 62, pp. 6470-6474.

19. Jeronimo, C., Nomoto, S., Caballero, O.L. et al. (2001), 'Mitochondrial mutations in early stage prostate cancer and bodily fluids', Oncogene Vol. 20, pp. 5195-5198.

20. Petros, J.A., Baumann, A.K., Ruiz-Pesini, E. et al. (2005), 'mtDNA mutations increase tumorigenicity in prostate cancer', Proc. Natl. Acad. Sci. USA Vol. 102, pp. 719-724.

21. Jessie, B.C., Sun, C.Q., Irons, H.R. et al. (2001), 'Accumulation of mitochondrial DNA deletions in the malignant prostate of patients of different ages', Exp. Gerontol. Vol. 37, pp. 169-174.

22. Fliss, M.S., Usadel, H., Caballero, O.L. et al. (2000), 'Facile detection of mitochondrial DNA mutations in tumors and bodily fluids', Science Vol. 287, pp. 2017-2019.

23. Bianchi, M.S., Bianchi, N.O. and Bailliet, G. (1995), 'Mitochondrial, DNA mutations in normal and tumor tissues from breast cancer patients', Cytogenet. Cell Genet. Vol. 71, pp. 99-103.

24. Tan, D.J., Bai, R.K. and Wong, L.J. (2002), 'Comprehensive scanning of somatic mitochondrial DNA mutations in breast cancer', Cancer Res. Vol. 62, pp. 972-976.

25. Tan, D.J., Liu, L.L., Wen, Y. et al. (2005), 'Heteroplasmy: A common phenomenon of mitochondrial genome mutations in human tumor tissues', Yi Chuan Vol. 27, pp. 44-48 [In Chinese]. 
26. Zhu, W., Qin, W., Bradley, P. et al. (2005), 'Mitochondrial DNA mutations in breast cancer tissue and in matched nipple aspirate fluid', Carcinogenesis Vol. 26, pp. 145-152.

27. Rosson, D. and Keshgegian, A.A. (2004), 'Frequent mutations in the mitochondrial control region DNA in breast tissue', Cancer Lett. Vol. 215, pp. 89-94.

28. Zhu, W., Qin, W. and Sauter, E.R. (2004), 'Large-scale mitochondrial DNA deletion mutations and nuclear genome instability in human breast cancer', Cancer Detect. Prev. Vol. 28, pp. 119-126.

29. Dani, M.A., Dani, S.U., Lima, S.P. et al. (2004), 'Less DeltamtDNA4977 than normal in various types of tumors suggests that cancer cells are essentially free of this mutation', Genet. Mol. Res. Vol. 3, pp. 395-409.

30. D310 refers to a deletion at bp 310 in the D-loop (also known as the control region and NCR in what is termed the homopolymer region, $\mathrm{C}$-stretch, $\mathrm{C}_{\mathrm{n}}$ and perhaps other designations as well. This region is characterised by a string of $\mathrm{C}$ nucleotides from bp $303-315 .^{53}$ In some mitochondrial lineages, there is a $\mathrm{T}$ at bp 310 as opposed to a $\mathrm{C}$. The exact use of the term D310 is somewhat ambiguous in the literature.

31. Isaacs, C., Cavalli, L.R., Cohen, Y. et al. (2004), 'Detection of LOH and mitochondrial DNA alterations in ductal lavage and nipple aspirate fluids from high-risk patients', Breast Cancer Res. Treat. Vol. 84, pp. 99-105.

32. Parrella, P., Xiao, Y., Fliss, M. et al. (2001), 'Detection of mitochondrial DNA mutations in primary breast cancer and fine-needle aspirates', Cancer Res. Vol. 61, pp. 7623-7626.

33. Alonso, A., Martin, P., Albarran, C. et al. (1997), 'Detection of somatic mutations in the mitochondrial DNA control region of colorectal and gastric tumors by heteroduplex and single-strand conformation analysis', Electrophoresis Vol. 18, pp. 682-685.

34. Polyak, K., Li, Y., Zhu, H. et al. (1998), 'Somatic mutations of the mitochondrial genome in human colorectal tumours', Nat. Genet. Vol. 20, pp. 291-293.

35. Habano, W., Sugai, T., Yoshida, T. and Nakamura, S. (1999), 'Mitochondrial gene mutation, but not large-scale deletion, is a feature of colorectal carcinomas with mitochondrial microsatellite instability', Int. J. Cancer Vol. 83, pp. 625-629.

36. Aikhionbare, F.O., Khan, M., Carey, D. et al. (2004), 'Is cumulative frequency of mitochondrial DNA variants a biomarker for colorectal tumor progression?', Mol. Cancer Vol. 3, pp. 30.

37. Habano, W., Nakamura, S. and Sugai, T. (1998), 'Microsatellite instability in the mitochondrial DNA of colorectal carcinomas: Evidence for mismatch repair systems in mitochondrial genome', Oncogene Vol. 17, pp. $1931-1937$.

38. Guleng, G., Lovig, T., Meling, G.I. et al. (2005), 'Mitochondrial microsatellite instability in colorectal carcinomas - Frequency and association with nuclear microsatellite instability', Cancer Lett. Vol. 219, pp. 97-103.
39. Pang, C.Y., Lee, H.C., Yang, J.H. and Wei, Y.H. (1994), 'Human skin mitochondrial DNA deletions associated with light exposure', Arch. Biochem. Biophys. Vol. 312, pp. 534-538.

40. Birch-Machin, M.A., Tindall, M., Turner, R. et al. (1998), 'Mitochondrial DNA deletions in human skin reflect photo - Rather than chronologic aging', J. Invest. Dermatol. Vol. 110, pp. 149-152.

41. Birch-Machin, M.A. (2000), 'Mitochondria and skin disease', Clin. Exp. Dermatol. Vol. 25, pp. 141-146.

42. LeDoux, S.P., Patton, N.J., Avery, L.J. and Wilson, G.L. (1993), 'Repair of $N$-methylpurines in the mitochondrial DNA of xeroderma pigmentosum complementation group D cells', Carcinogenesis Vol. 14, pp. $913-917$.

43. Pascucci, B., Versteegh, A., van Hoffen, A. et al. (1997), 'DNA repair of UV photoproducts and mutagenesis in human mitochondrial DNA', J. Mol. Biol. Vol. 273, pp. 417-427.

44. Croteau, D.L. and Bohr, V.A. (1997), 'Repair of oxidative damage to nuclear and mitochondrial DNA in mammalian cells', J. Biol. Chem. Vol. 272, pp. 25409-25412.

45. Sawyer, D.E. and Van Houten, B. (1999), 'Repair of DNA damage in mitochondria', Mutat. Res. Vol. 434, pp. 161-176.

46. Ray, A.J., Turner, R., Nikaido, O. et al. (2000), 'The spectrum of mitochondrial DNA deletions is a ubiquitous marker of ultraviolet radiation exposure in human skin', J. Invest. Dermatol. Vol. 115, pp. 674-679.

47. Durham, S.E., Krishnan, K.J., Betts, J. and Birch-Machin, M.A. (2003), 'Mitochondrial DNA damage in non-melanoma skin cancer', Br. J. Cancer Vol. 88, pp. 90-95.

48. Takeuchi, H., Fujimoto, A. and Hoon, D.S. (2004), 'Detection of mitochondrial DNA alterations in plasma of malignant melanoma patients', Ann. N Y Acad. Sci. Vol. 1022, pp. 50-54.

49. Deichmann, M., Kahle, B., Benner, A. et al. (2004), 'Somatic mitochondrial mutations in melanoma resection specimens', Int. J. Oncol. Vol. 24, pp. 137-141.

50. Poetsch, M., Petersmann, A., Lignitz, E. and Kleist, B. (2004), 'Relationship between mitochondrial DNA instability, mitochondrial DNA large deletions, and nuclear microsatellite instability in head and neck squamous cell carcinomas', Diagn. Mol. Pathol. Vol. 13, pp. $26-32$.

51. Suzuki, M., Toyooka, S., Miyajima, K. et al. (2003), 'Alterations in the mitochondrial displacement loop in lung cancers', Clin. Cancer Res. Vol. 9, pp. 5636-5641.

52. Chinnery, P.F. and Schon, E.A. (2003), 'Mitochondria', J. Neurol. Neurosurg. Psychiatry Vol. 74, pp. 1188-1199.

53. Andrews, R.M., Kubacka, I., Chinnery, P.F. et al. (1999), 'Reanalysis and revision of the Cambridge reference sequence for human mitochondrial DNA', Nat. Genet. Vol. 23, p. 147. 